\title{
TECNOLOGÍA ASISTIVA: PROPUESTA DE TERAPIA OCUPACIONAL PARA AUMENTAR EL DESEMPEÑO OCUPACIONAL DE UN USUARIO CON PARÁLISIS CEREBRAL
}

\author{
ASSISTIVE TECHNOLOGY: PROPURSE OF OCCUPATIONAL TERAPHY FOR INCREASE \\ OCCUPATIONAL PERFORMANCE OF USER WITH CEREBRAL PALSY
}

\section{Oscar Hernández Lanas ${ }^{1}$}

\begin{abstract}
Resumen:
El presente estudio de caso ha sido confeccionado para la obtención del título profesional de Terapeuta Ocupacional, en el marco del examen de pre-título exigido por la Universidad de Chile. Su objetivo es fomentar el uso de la tecnología asistiva como herramienta de intervención para la inclusión de personas con discapacidad, enmarcar las competencias que debe disponer el profesional en su utilización y fomentar su uso como complemento al enfoque tradicional de rehabilitación. Se expone el proceso de evaluación e intervención de un joven con diagnóstico de parálisis cerebral, con compromiso motor severo e intelectual leve, alto nivel de dependencia y escasas oportunidades de ejercer su autonomía. La experiencia es llevada a cabo en una institución de rehabilitación física infanto-juvenil de la Región Metropolitana, específicamente en una unidad de tecnología asistiva durante el año 2012. Engloba principalmente el proceso de evaluación y acceso al ordenador e instauración de un sistema inicial de comunicación aumentativa. Se concluye que la intervención realizada complementa los enfoques tradicionales de rehabilitación para fomentar la independencia, autonomía y desempeño ocupacional. Se visualiza la escasa sistematización de prácticas en Chile y se propone fomentar su desarrollo como un área de especialidad dentro de la Terapia Ocupacional.
\end{abstract}

\section{Palabras Clave:}

Tecnología Asistiva, Terapia Ocupacional, Parálisis Cerebral, Computador.

\begin{abstract}
:
This paper has been made in order to obtain the professional qualification of Occupational Therapist, an examination of prequalification required by Universidad de Chile. It aims to promote the use of assistive technology as a tool of intervention for the inclusion of people with disabilities, framing the competencies must have the professional in their use and encourage its use as a complement to the traditional approach of rehabilitation. It describes the process of assessment and intervention of a youth with cerebral palsy with severe motor and mild intellectual commitment, high dependency and limited opportunities to exercise their autonomy. The experience is held at an institution of child and adolescent physical rehabilitation of the metropolitan region, specifically assistive technology unit during 2012. It covers mainly the evaluation process and access to the computer and setting up an initial system of augmentative communication. Conclude that interventions complement traditional rehabilitation approaches to promote independence, autonomy and occupational performance. Displays the scarce systematization of practices in Chile intends to promote its development as a specialty area within Occupational Therapy.
\end{abstract}

Keywords:

Assistive Technology, Occupational Therapy, Cerebral Palsy, Computer.

1 Terapeuta Ocupacional, Licenciado en Ciencia de Ocupación Humana, Universidad de Chile. Contacto: (+569) 9140 10 35, oscarhernandezlanas@gmail.com. 


\section{INTRODUCCIÓN}

Históricamente la Terapia Ocupacional (T.O.) ha buscado diversas estrategias para mejorar las capacidades funcionales de los individuos con alguna discapacidad, con la finalidad de aumentar el desempeño ocupacional en actividades de autocuidado, escolaridad, trabajo, juego y ocio. Una de las formas que ha mostrado tener buenos resultados, ha sido la utilización de equipos adaptados conocidos como tecnologías asistivas (T.A.). La T.A. ha permitido amplificar las capacidades remanentes y modificar el microambiente de las personas con discapacidad, para ofrecer un aumento en la independencia durante la ejecución de una tarea, sin importar la edad o nivel funcional que disponga (Bain, 2005).

Desde sus inicios, la T.O. se ha comprometido a promover la autodeterminación y la inclusión de personas con discapacidad en todos los contextos, y es por esta razón que el Terapeuta Ocupacional ha utilizado la T.A. para facilitar a la persona el acceso a todos los ambientes, alcanzar una mayor autonomía y participación, además de aumentar el nivel de productividad e independencia (Atedisca Campony, 2012; Alcantud, 1999).

Una de las poblaciones que tiene limitado el ejercicio de su autonomía, su participación ocupacional, acceso a oportunidades y experiencias ambientales, son las personas con parálisis cerebral. La parálisis cerebral es causada por un daño cerebral que se produce antes, durante o después del nacimiento, provocando principalmente trastornos posturales y del movimiento, con los cuales pueden coexistir déficits visuales o auditivos, convulsiones, deterioro cognitivo, problemas del aprendizaje, disfagia y trastorno del lenguaje (Pelligrino, 1997 \& NINDS, 2001). Esta sintomatología complica la ejecución de actividades de alimentación, vestuario, higiene, como también la participación en experiencias lúdicas, académicas y sociales a lo largo del ciclo vital.

Desde la década de 1980, la T.A. se ha vuelto cada vez más importante en la vida diaria de la mayoría de las personas con discapacidad, especialmente para niños con parálisis cerebral (Anson, 1997). Estas tecnologías permiten a los individuos con un control motor limitado, facilitar tareas que se desempeñan con cierto grado de dificultad; y por otro lado, ganar la oportunidad de realizar actividades que de otro modo no sería posible su realización (Swinth \& Case-Smith, 1993).

En el presente estudio de caso se expone el proceso de evaluación e intervención de un joven con diagnóstico de parálisis cerebral, con compromiso motor seve- ro e intelectual leve, ausencia del lenguaje expresivo, alto nivel de dependencia y escasas oportunidades de ejercer su autonomía. La experiencia es llevada a cabo en una institución de rehabilitación física infanto-juvenil de la Región Metropolitana, específicamente en una unidad de tecnología asistiva durante el año 2012.

\section{Aspectos Bioéticos}

Para la elaboración del estudio de caso se solicita el consentimiento al cuidador principal y asentimiento al menor. Se busca con esta acción verificar la comprensión de la información entregada en relación a los objetivos de la intervención, las características de su participación y autorizar el uso y publicación de la información obtenida para fines académicos y científicos, con absoluta confidencialidad y respeto de la identidad de los participantes.

En el caso específico del asentimiento, se obtiene la autorización mediante la comunicación oral apoyada por material gráfico, utilizando un tablero de comunicación tradicional. La información se entrega de manera clara, adaptada a la edad mental y acorde al nivel sociocultural del participante.

La participación en este estudio no significó riesgo físico, psicológico ni emocional para las personas involucradas, como tampoco repercusiones para la institución en que se llevó a cabo la intervención.

\section{Descripción del Caso}

\section{Contexto Sociocultural y Marco Institucional}

La intervención se realiza en una institución que declara proveer asistencia a sus beneficiarios en los procesos de integración familiar, escolar, comunitaria, social y/o laboral, a través de programas médico-terapéuticos, psicosociales, educacionales, de desarrollo personal y recreacional (Blanco, 2006). Principalmente utiliza un enfoque de atención centrado en la asistencia, con énfasis en mejorar la funcionalidad, el autocuidado y la autovalencia (González, 2006).

Dentro de este contexto se conforma una unidad especializada en tecnología asistiva. Esta unidad tiene el propósito de potenciar el proceso de rehabilitación convencional entregado por la institución, a través de la implementación de T.A. y el desarrollo de nuevas habi- 
lidades en informática y comunicación. Busca que las personas con discapacidad puedan utilizar la tecnología para facilitar el proceso de aprendizaje, generar ambientes domiciliarios más accesibles, dar competitividad en el lugar de trabajo y aumentar su independencia en actividades de la vida diaria básica e instrumental (Cubillos et al. 2011).

Cada ingreso a esta unidad es de carácter individual por un período de 10 sesiones, las cuales son derivadas por el servicio interno o por demanda espontánea. El ingreso es evaluado por el equipo de la unidad, formado por Terapeutas Ocupacionales, Educadores Diferenciales, Fonoaudiólogos, Musicoterapeuta, Kinesiólogo y Diseñador Industrial. Al final del período se revalúa el cumplimiento de objetivos, pudiendo reingresar, egresar o derivar a otro programa dentro de la institución (Cubillos et al. 2011).

\section{Antecedentes Generales, Familiares y Socio- Históricos}

AGM es un adolecente de 15 años con diagnóstico de parálisis cerebral del tipo tetraparesia de predominio espástico con componentes atetósicos. Se observa falta de estabilidad proximal del tronco y cintura escapular, por lo cual sólo puede liberar sus miembros superiores parcialmente para realizar alcances; con un correcto posicionamiento mejora su funcionalidad a nivel distal. Ocupacionalmente es dependiente en todas las actividades de la vida diaria, pero logra manifestar necesidades, intereses de juego e interactuar con terceros con contacto social adecuado.

Se comunica principalmente con gestos y miradas; realiza movimientos de cabeza (sí/no) con nivel de respuesta inconsistente. Actualmente es incapaz de vocalizar a causa de un tono alto y fluctuante en la zona oral, con dificultad para coordinar la respiración al emitir sonidos.

Dentro de su historial médico-terapéutico ha recibido intervenciones de rehabilitación tradicional desde Kinesiología, Terapia Ocupacional, Fonoaudiología y Psicología. Se ha sometido a operaciones quirúrgicas para corregir luxación de cadera bilateral. Por escaso control motor y complicaciones musculoesqueléticas (escoliosis) utiliza silla de ruedas neurológica para permanecer en sedente.

Vive con ambos padres y es el menor de cuatro hermanos. Esta al cuidado principalmente de su madre, sin otra red de apoyo. Grupo familiar perteneciente al nivel socioeconómico medio-bajo.

En el ámbito escolar, asiste hasta los 9 años de edad a educación especial con intervalos de educación intrahospitalaria. Su asistencia al colegio ha sido irregular por intervenciones quirúrgicas y dificultades familiares. De estas últimas se destaca dinámica de sobreprotección, problemas de traslado al establecimiento e incompatibilidad horaria con trabajo de padres (nocturnos). Al momento de realizar la intervención los padres no han realizado ningún trámite para re-escolarizar al menor.

\section{Motivo de Consulta}

Ingresa a la unidad con el objetivo de generar una interface de acceso para el uso del computador con la finalidad que en el futuro, se implemente por esta vía un sistema de comunicación aumentativo alternativo (CAA).

\section{Evaluación de Terapia Ocupacional}

Se realiza una evaluación ocupacional basada en la revisión de ficha, observación del desempeño en el uso del computador y entrevistas al usuario y su familia. En una segunda instancia se aplican pautas específicas para el uso del computador, usando la "Pauta de Interface accesible de la Unidad de Tecnología Asistiva" y el método ALBOR (Acceso Libre de Barreras al Ordenador) (CEAPAT, 2012). Los resultados relevantes se organizan desde la perspectiva del modelo de desempeño ocupacional (MDO) y el modelo de actividad humana y tecnología asistida (HAAT).

\section{Resultados de la evaluación}

Como impresión general, se observa conectado al medio, con alta distractibilidad, de personalidad amigable y cooperadora.

a) Actividad: En este ítem se incluye: participación en áreas de desempeño, rutinas e intereses ocupacionales. AGM es dependiente en alimentación, higiene mayor y menor, uso WC, vestuario, trasferencia, control miccional e intestinal y desplazamientos. Actualmente no asiste a educación regular, observándose un desequilibrio ocupacional importante, 
con rutina centrada en actividades de tiempo libre y de mantención de salud. Su tiempo libre lo desempeña al interior de su hogar, principalmente en actividades pasivas como ver televisión en familia, escuchar radio o mirar actividades de terceros estableciendo en momentos interacción, práctica ocasionalmente social. Se encuentra familiarizado con el uso del computador, actividad placentera que motiva gran interés.

Con relación a la rutina de fin de semana participa en salidas con familia a lugares públicos y recibe visitas de familiares. En general, se desprende que su participación social es principalmente a nivel familiar, existiendo participación comunitaria en contextos protegidos.

b) Operador: Se incluye un análisis de habilidades de desempeño y factores del usuario, además de sus experiencias con equipos tecnológicos.

\section{- Funciones Corporales}

Neuromusculoesquelético y relacionado con el movimiento: Se observa en miembros superiores flexión y rotación interna de hombro, flexión de codo y muñeca, subluxación de pulgar reductible bilateral y dedos en cuello de cisne; hipertónicos (Ashworth 2), además de presentar acortamiento de musculatura flexora de antebrazo y brazo. En miembros inferiores se observa flexión bilateral de cadera en $90^{\circ}$ (con fijación quirúrgica de dicha articulación), rodillas con limitación de flexión de rodilla (logra hasta $90^{\circ}$ de flexión), pies en talo estable y ortejos en flexión; hipertónicos (Ashworth 3).

Voz y habla: Sólo es capaz de generar sonidos guturales, con aumento y disminución de volumen.

Sensoriales: Audición y visión normales.

Mentales: Comprende a nivel de enunciado verbal simple y de mediana complejidad. Es capaz de identificar objetos con la vista y comprender relaciones de causa y efecto relacionadas con el computador.

\section{- Habilidades de desempeño}

\section{- Habilidades motoras:}

Postura: Se observa falta de estabilidad proximal de tronco y cintura escapular. Logra mantener control de tronco y cabeza con fijaciones posturales a nivel distal; tendencia a generar mayor carga de peso a su hemicuerpo derecho. Logra posicionar sus MMSS a objetos que desea manipular.

Movilidad: Su nivel funcional más alto es sedente, por lo cual permanece en silla de rueda la mayor cantidad de tiempo y es trasladado por terceros. Sus alcances son principalmente en el plano sagital y horizontal, limitados a una distancia cercana a su cuerpo y que no supere la línea proyectada desde sus ojos. No es capaz de flexionar activamente el tronco y realizar giros.

Coordinación: Funcionalmente logra coordinar miembros superiores para tomar objetos de manera unimanual, con una base de apoyo amplia para no perder la estabilidad. Es capaz de tomar y liberar objetos a través de prehensiones dígito-palmares y pinza lateral, además de realizar empujes y tracciones. Realiza movimientos suaves y fluidos de poca presión.

En relación al esfuerzo sostenido y fuerza, no se observan alteraciones importantes.

\section{- Habilidades de proceso}

Atención: dificultad en mantener atención durante toda la actividad, se distrae con conversaciones de otras personas. Necesita de indicaciones verbales para terminar actividades.

Conocimiento: Tiene nociones generales sobre el uso del computador como elemento de entretención, no sabe utilizar el teclado y el mouse. No identifica partes del computador. Es capaz de manipular switch mecánicos. No es capaz de indagar para buscar más información.

Organización temporal: Es capaz de iniciar la tarea del uso del computador, de manera intuitiva presionando botones que dispone a su alcance, continúa acciones, genera secuencias de orden lógico (por ejemplo se acerca al computador, presiona el botón, genera una acción, espera, vuelve a presionar para continuar) y tiende a no terminar las actividades por inatención.

Organización espacio y objetos: No es capaz de organizar el espacio de trabajo para ocupar el computador, por lo cual los elementos de trabajos deben ser puestos de manera ordenada y a su alcance para ser utilizados.

Adaptación: Es capaz de responder órdenes simples a través de gestos de afirmación o negación. Tiene dificultad para anticipar problemas en la actividad, por lo cual reintenta conductas motoras positivas, en un nivel concreto. En caso de cometer un error, no es capaz de prevenir complicaciones. 


\section{- Habilidades de Comunicación e interacción}

Expresividad física: Sólo es capaz de orientar su cabeza hacia el contacto con terceros y mira cuando le hablan.

Intercambio de información: Se comunica a través de gestos vocálicos y kinésicos, con tablero de comunicación es capaz de señalar ítem y mantiene atención durante el barrido. Puede identificar a familiares y responde a órdenes simples sin gestos. Afirma y niega. No es capaz de solicitar necesidades generales o específicas. Saluda, se despide y agradece.

Relaciones: Actualmente no es capaz de mantener una relación apropiada con otras personas a través de una ocupación, principalmente por carecer un lenguaje oral o gestual que permita generar conversaciones o discusiones.

\section{- $\quad$ Experticia en AT}

Se clasifica como novato en la utilización del computador. Se espera que aprenda la utilización del computador como un sistema de comunicación aumentativa, con poca generalización del aprendizaje y un gran esfuerzo para poder controlar el equipo.

c) Contexto desempeño. En este ítem se incluye el medio ambiente físico, social, cultural e institucional. El usuario se desempeña principalmente al interior de su domicilio, en espacios comunitarios y en el instituto de rehabilitación. En estos ambientes debe interactuar con su familia nuclear, con el personal médico y en algunas ocasiones con desconocidos. Si se desea implementar algún dispositivo, este deberá ser aceptado por su círculo familiar cercano y ser apto para ser usado en ambientes ruidosos y con alta luminosidad. Dentro de posibles financiamientos, se puede solicitar la compra del equipo al Servicio Nacional de la Discapacidad (SENADIS). También puede optar por la confección de una alternativa de bajo costo.

d) Asistencia tecnológica: En este ítem se analiza principalmente el uso del computador.

Interface Hombre-Máquina: Se pretende generar una interface entre AGM y una computadora con la finalidad de instalar en esta última un sistema de CAA. Actualmente no es capaz de navegar y explorar con el mouse, ni utilizar el teclado convencional. Se decide explorar la mejor alternativa de acceso a través del método ALBOR, el cual es explicado en la sección de intervención.

Procesador: En la interface corresponde al software de CAA que mantendrá el control de datos y evitará posibles errores.

Respuesta: Como habilidad de salida se espera la función de sintetizador de voz, como función equivalente al lenguaje oral.

\section{Problemática del Paciente}

Por correspondencia al ciclo vital, AGM debería desempeñar el rol de estudiante y comenzar la participación social en grupos de amigos y dispositivos sociales de su interés. Estos roles no son ejercidos, principalmente por su compromiso motor severo (dificultad en movilidad y dependencia total en actividades de la vida diaria) y por dificultad presente para relacionarse y comunicarse con desconocidos.

Se opta intervenir desde un enfoque rehabilitador/ compensador, por lo cual se propone trabajar la problemática desde el enfoque de tecnología asistiva. Se realiza consulta a usuario y su familia sobre objetivo a trabajar. Madre reporta que le gustaría que AGM sea capaz de opinar y relacionarse con desconocidos, mejorar su autonomía frente a la asistencia que recibe; mientras que AGM manifiesta el deseo de utilizar una computadora, por ser divertida y siente curiosidad por utilizarla como sistema de comunicación. Se le informa que los resultados serán dependientes del nivel de experticia que adquiera con el dispositivo.

\section{Modelo de Intervención}

En el presente caso, la intervención se fundamenta en teorías derivadas del comportamiento ocupacional, el modelo de desempeño ocupacional (MDO) y modelo de actividad humana y tecnología asistiva (HAAT).

La teoría del comportamiento ocupacional nos permite comprender al ser humano como un ser ocupacional, que participa todo el tiempo en ocupaciones, que Ilenan de significado su vida, permite cubrir necesidades de mantenimiento personal, expresión y logro. Con el uso terapéutico de estas ocupaciones, se puede impactar en la calidad de vida y bienestar (Florey, 1969, Really, 1966, 1974 en Barret \& Kielhofmer, 2005). En el presente caso, el usuario no es capaz de participar 
de forma independiente en ninguna ocupación, por lo cual no tiene la posibilidad de cubrir sus necesidades de mantenimiento personal, expresión y logro, lo que impacta en su calidad de vida y bienestar.

A pesar de esto, se observa que muestra curiosidad e intenta explorar roles primarios dentro de la sociedad, principalmente el de jugador y miembro de familia. Es por esta razón que es vital que se realice un análisis de su Desempeño Ocupacional, por lo cual se selecciona el modelo de desempeño ocupacional como modelo de referencia (Figura $\mathrm{N}^{\circ} 1$ ).

Figura $\mathrm{N}^{\circ} 1$

Modelo de Desempeño Ocupacional (Polonio, 2001)

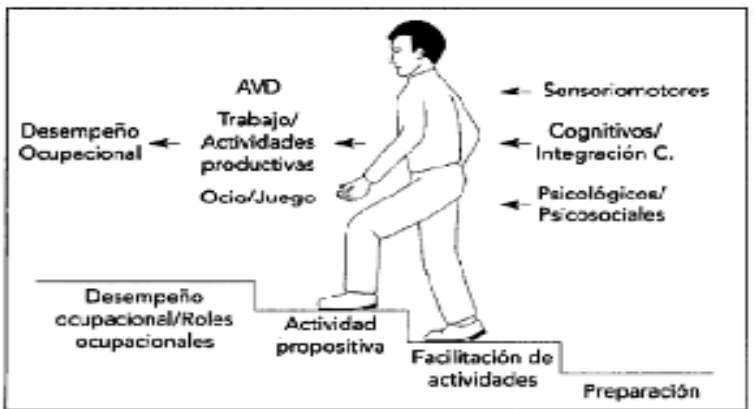

Este modelo permite analizar las áreas de desempeño (AVD-B e I, actividades productivas y actividades de ocio y juego), las habilidades del desempeño (motoras, sensoriales y praxis, de procesamiento y de regulación emocional) y los contextos de la ejecución (aspectos temporales y ambientales). Como elemento central analiza el desempeño ocupacional, entendido como la capacidad para ejecutar aquellas tareas que hacen posible desarrollar los roles ocupacionales de manera satisfactoria y apropiada en relación con: edad del individuo, cultura y entorno. El desempeño ocupacional requiere aprendizaje y experiencia en la realización del rol, un nivel de desarrollo específico para el desempeño de las tareas y la utilización de todos los componentes del desempeño. Los déficits en las experiencias de aprendizaje, de las habilidades de desempeño y/o del espacio vital, pueden producir limitaciones en el desempeño ocupacional (Polonio, 2001).

El modelo plantea que el rol del Terapeuta Ocupacional consiste, en líneas generales, en ayudar a alcanzar la máxima independencia posible en las áreas de desempeño ocupacional y a reasumir o proponer roles. Plantea un continuum terapéutico, en el cual las etapas del tratamiento se solapan o se dan simultáneamente. Este continuum no sigue una progresión escalonada estrictamente, sino que sigue una progresión lógica que va desde la dependencia en la actividad hasta la reasunción de los roles vitales. Las etapas son las siguientes: (1) métodos preparativos para la actividad; (2) métodos facilitadores y coadyuvantes, (3) actividad propositiva y por último (4) desempeño ocupacional y roles ocupacionales. Con estas acciones se espera generar un dominio de la actividad, donde la persona sea eficaz en el uso de su tiempo, energía y recursos, lograr los objetivos que desea y obtenga una respuesta de aprobación de su medio cercano. (Schultz \& Schkade, 1997).

Por otro lado, se apoyará la intervención con el uso de Tecnología Asistiva. Entenderemos como tecnología asistiva (T.A). a "cualquier objeto, equipo, sistema, producto, adaptación y servicio cuyo propósito es el de suplir, aumentar, mantener o mejorar las capacidades funcionales de los individuos con discapacidades" (Roca; Roca \& Del Campo, 2001). Los servicios de tecnología incluyen la ayuda directamente a una persona con discapacidad en la selección, adquisición o uso de tecnología asistiva. Dentro de las etapas que el terapeuta ocupacional presta servicio, se encuentran: la evaluación de las necesidades y capacidades del individuo, selección de artículos de T.A. de acuerdo a las necesidades del individuo, adquisición, capacitación y/o entrenamiento de la misma (Bain, 2005).

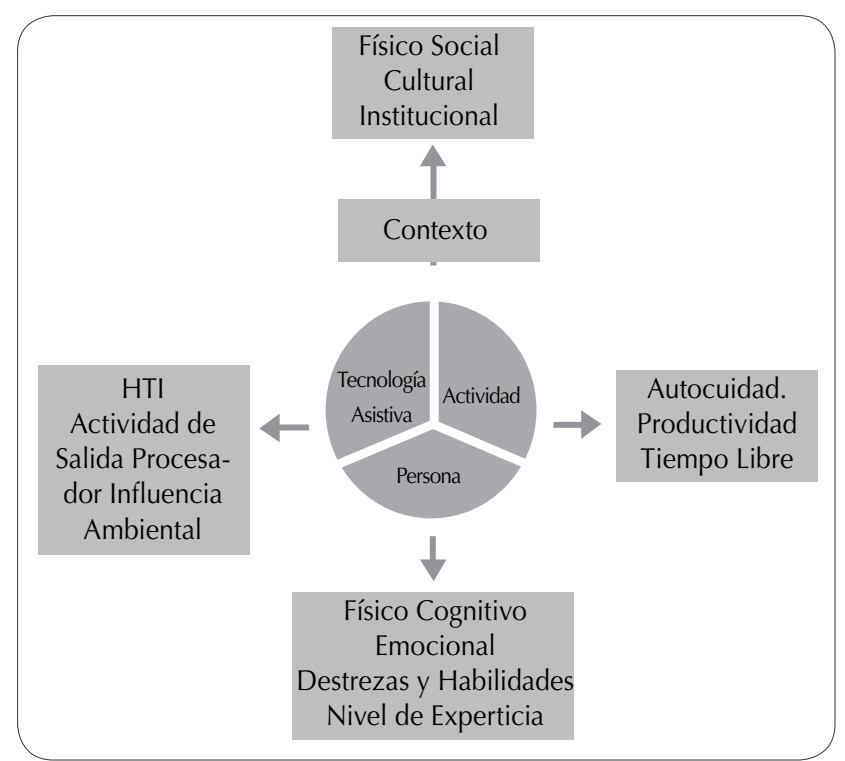

Para poder prestar estos servicios, se propone la utilización de Modelo HAAT (Figura $\mathrm{N}^{\circ} 2$ ). El HAAT es un modelo interdisciplinario que analiza la interacción 
que tiene la persona al utilizar una tecnología asistida en una actividad de su interés, además de analizar su desempeño en relación al contexto. Tiene como objetivo el poder prescribir una T.A. que se ajuste a las necesidades de la persona, y a través de su uso, generar resultados funcionales sin tener que intervenir la capacidad de la persona o en sus discapacidades. El modelo considera un sistema de cuatro componentes integrados e interdependientes: la persona, las tareas y objetivos que ésta necesita cumplir (actividad), los dispositivos (T.A.) y el ambiente. Cada componente debe ser evaluado en el contexto de los otros, seleccionado sobre la base del modo en que se conecta con los demás y aplicado según cómo interactúa con éstos. (Cook \& Hussey 1995).

Por otro lado, el uso de T.A. como herramienta terapéutica se basa principalmente en teorías de las perspectivas de rehabilitación, específicamente de los marcos de referencia de la rehabilitación (adaptación de la tarea y modificación ambiental) y biomecánica (simplificación del trabajo y principios de conservación de energía) y de teorías derivadas del aprendizaje (Giuffrida \& Neistadt, 2005).
Para este caso, desde la perspectiva de rehabilitación se destaca que la intervención terapéutica debe estar dirigida a modificar o adaptar las características contextuales y las demandas de la tarea para que apoyen el desempeño en el contexto (Blesedell, 2005). Estas adaptaciones deben considerar al usuario y sus contextos como elementos singulares y dinámicos que se influyen recíprocamente (Dunn; McClain; Brown \& Youngstrom, 2005). Desde este postulado, se considera la asistencia tecnológica como una estrategia de compensación que permite adaptar la tarea con su uso (simplificación de la tarea y ahorro energético) y modificar el microambiente de la persona, con la finalidad de recobrar la independencia sin tener que remediar el deterioro subyacente. Se debe analizar además los niveles de supervisión, asistencia disponible y expectativas de desarrollo con el dispositivo (Dutton, 1995 en Seidel, 2005).

Por último, se debe examinar el proceso de enseñanza-aprendizaje, ya que al implementar cualquier modificación a la actividad o el ambiente, el usuario necesitará tener un nivel mínimo de habilidades cognitivas que le permita aprender, aplicar y trasferir su uso a todas las ocupaciones realizadas cotidianamente (Toglia, 1998).

\section{Propuesta de Intervención}

\section{Objetivos Generales, Específicos e Indicadores de Logros}

\begin{tabular}{|c|c|c|}
\hline Objetivos Generales & Objetivos Específicos & Indicadores de Logro \\
\hline \multirow[t]{2}{*}{$\begin{array}{l}\text { Mejorar el desempe- } \\
\text { ño ocupacional en el } \\
\text { uso de computador }\end{array}$} & $\begin{array}{l}\text { Lograr que el usuario maneje un } \\
\text { dispositivo que genere las funcio- } \\
\text { nes del mouse (botón derecho, } \\
\text { izquierdo, arrastre y doble clic) }\end{array}$ & $\begin{array}{c}\text { Que el usuario sea capaz de: } \\
\text { Hacer un clic con botón izquierdo } \\
\text { Hacer un clic con botón derecho } \\
\text { Hacer un doble clic } \\
\text { Arrastrar iconos } \\
\text { No hacer pulsaciones no deseadas al mover } \\
\text { Activar la función frente a un estímulo } \\
\text { Mantener pulsación durante el tiempo que requiera el soft- } \\
\text { ware. }\end{array}$ \\
\hline & $\begin{array}{l}\text { Lograr que el usuario maneje una } \\
\text { alternativa tecnológica para la na- } \\
\text { vegación con el cursor del mouse. }\end{array}$ & $\begin{array}{c}\text { Que el usuario sea capaz de: } \\
\text { Navegar por la pantalla y seleccionar iconos } \\
\text { Arrastrar iconos }\end{array}$ \\
\hline
\end{tabular}




\begin{tabular}{|c|c|c|}
\hline Objetivos Generales & Objetivos Específicos & Indicadores de Logro \\
\hline \multirow[b]{2}{*}{$\begin{array}{l}\text { Mejorar el desempe- } \\
\text { ño ocupacional en } \\
\text { conversaciones con } \\
\text { su familia nuclear }\end{array}$} & $\begin{array}{c}\text { Evaluar y seleccionar tecnología } \\
\text { asistiva idónea para implementar } \\
\text { CAA }\end{array}$ & $\begin{array}{l}\text { Evaluar habilidades de desempeño del usuario } \\
\text { Proponer y probar posibles soluciones } \\
\text { Seleccionar sistema CAA para el entrenamiento } \\
\text { Derivar a fonoaudiología para su entrenamiento. }\end{array}$ \\
\hline & $\begin{array}{l}\text { Lograr comunicación aumentativa } \\
\text { - alternativa de necesidades bási- } \\
\text { cas de manera satisfactoria. }\end{array}$ & $\begin{array}{c}\text { Que el usuario sea capaz de: } \\
\text { Relacionarse con personas familiares a través del } \\
\text { uso del dispositivo } \\
\text { Interactuar con personas familiares a través del uso } \\
\text { del dispositivo. } \\
\text { Entregar información de sus necesidades básicas a personas } \\
\text { familiares a través de uso del dispositivo } \\
\text { Que la familia: } \\
\text { Conozca características del sistema seleccionado. } \\
\text { Interactúe con el usuario y el equipo prescrito. }\end{array}$ \\
\hline
\end{tabular}

\section{Programa de Actividades}

Se realizan 14 sesiones, de carácter individual en un ambiente protegido. Durante la intervención se cuenta con la cooperación constante de la madre del usuario. Se aplican evaluaciones específicas de T.A. y T.O., observación estructurada del uso del computador, aplicación del método ALBOR, entrenamiento de soluciones tecnológicas, revaluación, alta y entrega de indicaciones.

La intervención se centra en dos soluciones tecnológicas, por un lado generar una interface de acceso a un computador, por ser una herramienta versátil que podría apoyar el proceso educativo, configurarse como sistema de CAA o fomentar tiempo libre y participación social; y por otro, seleccionar y entrenar algún dispositivo de comunicación aumentativa que utilice mensajes grabados y de fácil activación que permita una comunicación más precisa con su entorno cercano.

Como metodología de trabajo se realiza principalmente una evaluación detallada de las necesidades y capacidades del individuo, para posteriormente seleccionar y entrenar posibles soluciones tecnológicas que respondan a la problemática detectada en la evaluación. Una vez consolidado el aprendizaje y teniendo la solución definitiva, se debe guiar en el proceso de adquisición de la T.A y luego entrenar en el contexto real de uso.

Para la evaluación y selección de la interface de acceso al computador, se utiliza el método ALBOR (CEAPAT, 2012). El método Albor consta de un sistema inteligente capaz de asesorar a los profesionales, tanto en la evaluación de capacidad de acceso al ordenador, como en la elección de las adaptaciones tecnológicas más adecuadas para posibilitar dicho acceso.

El sistema funciona en Internet, a través de cualquier navegador comercial, y apoya las decisiones con enlaces a información relevante relacionada con las adaptaciones sugeridas en cada caso. El sistema inteligente se encarga de identificar las aptitudes del usuario mediante un cuestionario dividido en cinco bloques: antecedentes del usuario y tarea a realizar, aptitudes cognitivas, físicas, visuales y auditivas. Seguidamente, se genera un modelo de la persona evaluada que sirve de entrada para el agente inteligente, quien generará una serie de recomendaciones que permiten determinar las ayudas técnicas más adecuadas para el caso (Montecino \& Carmona, 2005). Este resultado se debe contrastar con el razonamiento y experiencia clínica del Terapeuta Ocupacional que realiza la intervención.

Este método permite realizar una evaluación coherente con el modelo HAAT, ya que interrelaciona las capacidades de la persona con la actividad, y recomienda el tipo de tecnología asistiva según el contexto de uso. Además se debe considerar un análisis de postura en su dinámica movilidad versus estabilidad.

Durante las sesiones se procura que el usuario mantenga una postura adecuada en sedente, con acceso frontal al puesto de trabajo y elementos a manipular dentro de su rango de alcance. Se procura la mantención de una postura relativamente erguida, con el 
tronco estabilizado para permitir la función distal del miembro superior. La estabilidad del tronco se logra con una correcta estabilización de la pelvis y uso de un soporte dorsal. Para generar mayor estabilidad, se apoya postura con una mesa con escotadura y utilización de un vástago en el miembro izquierdo. Estas modificaciones permiten favorecer una acción sinérgica en cadena cerrada hacia la extremidad contralateral, brindando estabilidad proximal y movilidad distal con mayor precisión.

Del proceso de evaluación, se desprende que la navegación del cursor por la pantalla se podría dominar a través del uso de:

- Control cefálico, utilizando Smart $\mathrm{Nav}^{2}$ o navegación vía cámara web (Figura №3).

- Programa de selección de movimiento del cursor a través de temporizador activado por pulsadores (por ejemplo INTIC), siempre y cuando logre incrementar períodos de atención frente a la actividad.

- Sistema Touch, con ayuda técnica que permita el aislamiento de un dedo.

- $\quad$ Mouse activado por cinco pulsadores.

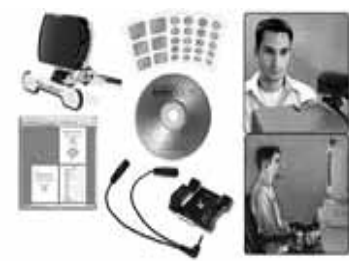

Figura No3 Smart Nav

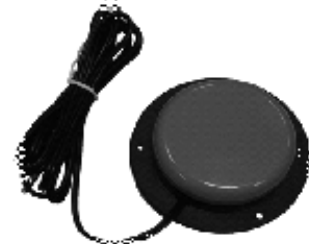

Figura N4: Pulsador

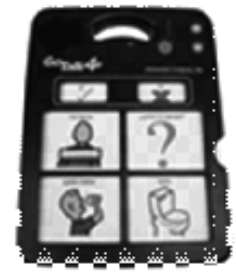

Figura N5: Tablero CAA
La función de clic (derecho e izquierdo) del mouse se logra a través de un pulsador mecánico (Figura $N^{\circ}$ 4). Se sugieren los siguientes puntos de control (del más eficiente al menos eficiente):

- Movimiento de mano derecha de adelante hacia atrás.

- Movimiento de codo derecho hacia atrás.

- Movimiento de mano derecha de arriba hacia abajo.

- Movimiento de cabeza hacia atrás (región occipital)

- Movimiento de cabeza de arriba hacia abajo (control mentoniano) (presenta sialorrea con esta opción)

Responde frente a estímulos pertinentemente y es capaz de mantener pulsado el botón durante un tiempo óptimo para la función de arrastre. La mayor dificultad en el usuario es la presencia de movimientos involuntarios de sus manos y la asociación de estos con movimientos de cabeza, se proyecta que lograría manejar de 2 a 3 pulsadores con sus manos siempre que sean visualmente diferentes. Por falta de educación formal, es difícil instaurar escritura en teclado por lo cual no se realiza entrenamiento en esta área.

Se realiza entrenamiento con cada uno de los dispositivos seleccionados, a través de juegos, programas y videos disponibles en internet. Se utiliza entrenamiento fraccionado en tareas parciales.

Por otro lado, con los avances en esta área se puede implementar un sistema de comunicación aumentativo, de dos respuestas (Sí/NO) (Figura $N^{\circ} 5$ ), el cual es activado por dos pulsadores de diferente color, con movimiento hacia adelante y atrás con miembro superior derecho. Se realiza juego con madre y se capacita en el tipo de preguntas que se debe realizar. Además se podría instalar un software de comunicación (Plaphons ${ }^{3}$ ) en algún dispositivo convencional como celular, tablet o notebook, pudiendo establecer una interface de comunicación. Esta opción aún no es válida por el poco dominio del computador; se sugiere seguir entrenando en su uso en el próximo ingreso.

Software gratuito que permite crear tableros de comunicación aumentativa. Incluye sintetizador de voz.
Dispositivo tecnológico que permite controlar el cursor de cualquier computador sin utilizar las manos, sustituyendo la función del mouse y teclado. 


\section{Resultados de la Intervención:}

Al término del ingreso, es posible señalar que se cumplen parcialmente los objetivos planteados. Como resumen, el usuario es capaz de hacer clic y doble clic con pulsador, los cuales es capaz de activar frente a estímulos, además de navegar con el cursor a través del Smart Nav. Ambas tareas no las realiza con la precisión necesaria para manejarla de manera independiente, por lo cual se sugiere seguir entrenando con ellas. Como logra manejar dos pulsadores al mismo tiempo, es posible implementar un dispositivo básico de comunicación, que maneje respuestas dicotómicas (positivo / negativo). Se desprende además, que la utilización de computador de manera autónoma no es posible, pero sí un entrenamiento en un software específico, donde deba asociar un switch a respuestas concretas. Una propuesta podría ser el uso del software Plaphons con secuencias básicas de interacción (saludos sociales, rutinas de alimentación, vestuario e higiene), siempre y cuando domine la navegación con el mouse en conjunto con la selección de iconos.

Una vez consolidados estos elementos, se debe guiar a la familia en solicitar la A.T. al SENADIS y posteriormente recibir entrenamiento por Fonoaudiología. Si evoluciona positivamente, se deberá entrenar el sistema de comunicación aumentativa en contextos naturales y con ello intentar que trasfiera el aprendizaje. Se debe además realizar una intervención familiar para que el usuario sea integrado a un sistema escolar, ya que al participar en este contexto serviría para estimular el área social y cognitiva, además de ejercer sus derechos y brindar nuevos desafíos.

\section{CONCLUSIÓN}

La experiencia compartida en este trabajo pretende sembrar la inquietud y la necesidad de trabajar y conocer más sobre el desarrollo de las asistencias tecnológicas aplicadas en la práctica de Terapia Ocupacional. El estudio presentado tiene una perspectiva a mediano y largo plazo para poder implementar un sistema de comunicación aumentativa alternativa, para ser usado regularmente en sus contextos cotidianos. Como se ha descrito anteriormente, teóricamente el uso de tecnología es una buena herramienta de intervención, ya que genera una oportunidad real de aumentar las habilidades de la personas y disminuir las barreras que presenta el contexto, permitiendo la participación en actividades que de otra forma no serían posibles. Durante la implementación del programa, se devela que esta área de Terapia Ocupacional necesita de cierta calificación o especialidad, sobre todo conocimientos de equipos, software, costos para su adquisición y conocer políticas gubernamentales de ayuda en su obtención, además de las competencias tradicionales que nos exige la profesión; el determinar el perfil ocupacional del usuario y un análisis de su desempeño.

Actualmente en Chile existen algunas experiencias y sistematizaciones sobre su aplicación, pero aún estamos lejos en el desarrollo de esta temática en relación a países como España y Estados Unidos; es por esta razón que es deber de nuestra profesión, empezar a generar posibles evaluaciones y guías de intervención acorde a la realidad nacional. A pesar de esto, en Chile se están implementando centros con esta especialidad, desarrollando respuestas individuales y colectivas a personas que viven con alguna discapacidad, pero aún falta mucho por desarrollar y luchar, buscando no sólo una oportunidad para algunos de mejorar sus condiciones de vida, sino que llegue a ser un derecho y que este se concrete en la práctica.

En cuanto a elementos prácticos observados, es una estrategia que complementa los enfoques tradicionales, que exige la implementación de equipos interdisciplinarios para comprender todas las aristas de la persona afectada. Se visualiza como una opción real para manejar el desempeño, pero al igual que la mirada de rehabilitación tradicional, muchas veces no se configura como la forma más rápida y eficiente para obtener resultados.

En cuanto a los resultados obtenidos, es posible señalar que la utilización del sistema de comunicación y un acceso al computador inicial, ha mejorado el sentido de competencia del usuario, permitiendo que aumente su independencia y sobre todo en la autonomía al momento de decidir en temas de asistencia, poder responder a preguntas simples, intentar aportar en una conversación y sobre todo manifestar ciertos deseos. Como se ejemplifica en este caso, al usar de dispositivos de tecnología asistiva damos la oportunidad a personas con discapacidad de participar en ocupaciones acorde a su edad, fomentar y estimular su desarrollo, además de permitir ejercer sus derechos al interior de la sociedad.

En definitiva, utilizar este enfoque de intervención por terapeutas ocupacionales puede ser efectivo y provechoso para el desempeño de la persona a intervenir, pero aún hay muchos desafíos en el área y se invita a los profesionales seguir investigando y probando esta 
estrategia, además de compartir sus experiencias tanto dentro y fuera del ámbito profesional.

\section{ReferenCiAs BibliográficAs}

Alcantud, F. (1999). "Las tecnologías de ayuda: un modelo de interverción". Conferencia marco en las I Jornadas Nacionales de ESAAC. Vitoria. Disponible en:<http://care.org.ar.elserver. com/sitio/images/stories/Doc\%20Utiles/Tecnologias_de_ Ayuda.pdf $>$

Atedisca Company (2012). ¿Qué es Tecnología Asistiva? Recuperado el 30/12/12 de <atedisca-wwwayudastecnologicascom.blospost.com>

Anson, D. (1997). "Alternative computer access. A guide to selection". Philadelphia: Davis.

Bain, B. (2005). "Tecnología de asistencia en terapia ocupacional" en Crepeu, E.; Cohn, E. \& Schell, B. "Willard \& Spackman. Terapia Ocupacional”. (pág. 659 - 675). 10ª edición. Buenos Aires, Argentina: Editorial Médica Panamericana.

Barret, L. \& Keielhofner, G. (2005). "Generalidades sobre el Comportamiento Ocupacional". En Crepeu, Cohn, \& Schell, Willard \& Spackman. Terapia Ocupacional (págs. 209 - 212). $10^{a}$ edición. Buenos Aires: Médica Panamericana.

Blanco, R. (2006): "Aspectos generales de la rehabilitación". En Sociedad Pro Ayuda al Niño Lisiado, Teletón. Enfermedades invalidantes de la infancia: enfoque integral de Rehabilitación, Vol. II. IVROS impresores.

Blesedell, E. (2005). "Análisis de la ocupación y la actividad: una forma de pensar sobre el desempeño ocupacional", en Crepeu, E. Cohn, E. \& Schell, B. "Willard \& Spackman Terapia Ocupacional". (10ª edición). ). Buenos Aires, Argentina: Editorial Médica Panamericana.

CEAPAT (2012). "Guía usuario Método Albor". Disponible en: $<$ http://herramientas.educa.madrid.org/albor/guia/guia.html>

Cook, R. \& Hussey, S. (1995). "Assitive Technologies: Principles and practice". St. Louis: Mosby.

Cubillos, R.; Barriga K.; Valenzuela R.; Cortés A.; Caro P. \& Siques C. (2011). "Manual de procedimientos Unidad de Tecnología Asistiva". Teletón Santiago. Documento no publicado.

Dunn, W.; McClain, L.; Brown, C. \& Youngstrom, M. (2005). "Ecología del desempeño humano". En Crepeu, Cohn, \& Schell, Willard \& Spackman Terapia Ocupacional (págs. 223 - 227). $10^{a}$ edición. Buenos Aires: Médica Panamericana.

Giuffrida, C. \& Neistadt, M. (2005). "Consideraciones generales de la teoría del aprendizaje". En Crepeu, Cohn, \& Schell, Willard \& Spackman. Terapia Ocupacional (págs. 253 - 257). 10ª edición. Buenos Aires: Médica Panamericana.
González, M. (2006). "Rehabilitación Infantil: Un desafío permanente". En Sociedad Pro Ayuda al Niño Lisiado, Teletón. Enfermedades invalidantes de la infancia: enfoque integral de Rehabilitación, Vol. II. IVROS impresores.

Montecinos, M.; Carmona, L. (2005). "Tecnologías para el acceso al ordenador". En Montecinos, M.; Carmona, L., et al. (2005) .Tecnologías de ayuda en contextos escolares". Servicio de Publicaciones y Estadística, España.

Pelligrino, L. (1997). "Cerebral Palsy", en Batshaw, M. "Childrem with disabilities" (pág. 499 - 528). Baltimore: Brookes National Institute of Neurological Disorders and Stroke [NINDS]. (2001). "Cerebral Palsy: Hope through research?". Recuperadado el 20/12/12 de <www.nimh.gov.Accesseded>

Polonio, B. (2001): "Modelo de desempeño ocupacional de la Asociación Americana de Terapia Ocupacional (AOTA)", en Polonio, B.; Durante, P. \& Noya, B. "Conceptos Fundamentales de Terapia Ocupacional" (pág. 104 - 109): Buenos Aires, Argentina: Editorial Médica Panamericana.

Roca, J.; Roca, J. \& Del Campo, M. (2001). “De las ayudas técnicas a la tecnología Asistiva". Diponible en <www.tecnoneet.org/ docs/2004/2-12004.pdf>

Schultz, S. \& Schkade, J. (1997): "Adaptación Ocupacional". En Crepeu, Cohn, \& Schell, Willard \& Spackman. Terapia Ocupacional (págs. 220 - 223). 10ª edición. Buenos Aires: Médica Panamericana.

Switnth, Y. \& Case-Smith, (1993). "Assitive technology in early intervention: Theory and practice" en Case-Smith J. "Pediatric occupational therapy and eartly intervention" (pág. 342 - 368). Boston: Andover Medical.

Seidel, A. (2005). "Perspectivas de rehabilitación". En Crepeu, Cohn, \& Schell, Willard \& Spackman Terapia Ocupacional (págs. 235 - 240). 10 a edición. Buenos Aires: Médica Panamericana.

Toglia, J. (1998). "A dynamic interactional model to cognitive rehabilitation". En Katz, N. "Cognition an occupational therapy" (pág. 5 -50). Bershesda, MD: American Occupational Therapy Association. 